\title{
Utilisation of cellulose microcapillary tubes as a model system for culturing and viral infection of mammalian cells
}

Eudri Venter ${ }^{1}$, Christiaan F. van der Merwe ${ }^{2}$, and Vida van Staden ${ }^{1}$

1. Department of Genetics, University of Pretoria, Pretoria, 0002, South Africa.

2. Laboratory for Microscopy and Microanalysis, University of Pretoria, Pretoria, 0002, South Africa.

\section{Running title:}

Cellulose microcapillary tubes for cell culturing and virus infection

Correspondence to be sent to:

Vida van Staden

Department of Genetics

University of Pretoria

Private Bag X20

Hatfield

0028

South Africa

E-mail: vida.vanstaden@up.ac.za

Telephone: +27 124203257

\section{ABSTRACT}

Cryofixation by high-pressure freezing (HPF) and freeze substitution (FS) gives excellent preservation of intracellular membranous structures, ideal for ultrastructural investigations of virus infected cells. Conventional sample preparation methods of tissue cultured cells can however disrupt the association between neighbouring cells or of viruses with the plasma membrane, which impacts upon the effectiveness whereby virus release from cells can be studied. We established a system for virus infection and transmission electron microscopy preparation of mammalian cells that allowed optimal visualisation of membrane release events. African horse sickness virus (AHSV) is a non-enveloped virus that employs two different release mechanisms from mammalian 
cells, i.e. Iytic release through a disrupted plasma membrane and a non-lytic buddingtype release. Cellulose microcapillary tubes were used as support layer for culturing Vero cells. The cells grew to a confluent monolayer along the inside of the tubes and could readily be infected with AHSV. Sections of the microcapillary tubes proved easy to manipulate during the HPF procedure, showed no distortion or compression, and yielded well preserved cells in their native state. There was ample cell surface area available for visualisation, which allowed detection of both types of virus release at the plasma membrane at a significantly higher frequency than when utilising other methods. The consecutive culturing, virus infection and processing of cells within microcapillary tubes therefore represent a novel model system for monitoring intracellular virus life cycle and membrane release events, specifically suited to viruses that do not grow to high titres in tissue culture.

\section{Keywords}

High-pressure freezing, African horsesickness virus, transmission electron microscopy

\section{INTRODUCTION}

Ultrastructural studies of virus infected cells allow characterisation of the dynamic processes of virus entry, intracellular transport, cellular response and morphological changes resulting from viral infection, and assembly and release of progeny viruses. This requires in situ analysis of biological material, with preservation of the cellular fine structure and association of neighbouring cells as close as possible to the native state. The cryopreservation of cells by high-pressure freezing followed by freeze-substitution (HPF-FS) minimises artefacts induced by aldehyde fixation or ice crystal damage, and is specifically suited for the high resolution study of intracellular membranous structures (Studer et al., 2001). High-pressure freezing (HPF) can be used to efficiently immobilize thick suspensions or pellets of yeast or mammalian cells, or even small pieces $(<0.5 \mathrm{~mm}$ ) of plant or animal tissue (Morphew and Mclntosh, 2003). For transmission electron microscopic (TEM) studies of viruses in tissue culture, adherent cell lines are normally cultured as a monolayer, infected, and harvested at the desired 
time post infection. Both physical or enzymatic removal of cells from their growth support may result in disruption of the cellular architecture (Monaghan et al., 2004). In addition, exposure to the shear and centripetal forces of a centrifugation step can further distort the natural arrangement of the cells or associations of viruses with the cell surface. The concentrated cell sample can be loaded directly into a specimen carrier suitable for the HPF apparatus. Alternatively cellulose microcapillary tubes filled with cells have been successfully used as mini-containers for transferring samples into the HPF specimen holder (Hohenberg et al., 1994; Muller-Reichert et al., 2003; Rieger et al., 1997; Studer et al., 2001). The $200 \mu \mathrm{m}$ inner diameter of the microcapillary tubes falls within the sample thickness limits for optimal fixation by HPF (Studer et al., 1992), and gives high yields of vitrified biological material (Hohenberg et al., 1994; MullerReichert et al., 2003; Rieger et al., 1997; Studer et al., 2001).

Ultrastructural studies of virus infected cells prepared by HPF-FS have been used extensively for a wide variety of viruses (Fontana et al., 2010; Monaghan et al., 2004; Reichelt et al., 2011; Suhy et al., 2000; Wild et al., 2002), however the majority of these studies focussed on description of intracellular viral lifecycle events. If one is interested in visualising membrane or receptor bound viruses at the cell surface, in the process of entering into or exiting from a cell, these events could well be disrupted by the mechanical handling of cells prior to HPF. Therefore, in instances where the monolayer culture serves as a model system for a biological process, the cells under study have to be grown upon a support layer that is amenable to co-processing through the HPF-FS protocol. Previous reports describe the use of membrane filters (Morphew and Mclntosh, 2003), sapphire disks (Reipert et al., 2004), copolymer films (Jimenez et al., 2006), or dextran microcarriers (Hagen and Grünewald, 2008) for this purpose. In this study we investigated the use of cellulose microcapillary tubes for the consecutive culturing of mammalian cells, infection by virus, and processing by HPF-FS for electron microscopy.

African horse sickness virus (AHSV) was used as model virus for this study, as it employs two different mechanisms of virus release from the same cell type. AHSV is an 
insect borne virus, belonging to the family Reoviridae and genus Orbivirus, transmitted by Culicoides hematophagous midges. It causes African horse sickness (AHS), a listed disease of the World Organisation for Animal Health (OIE) and one of the most lethal of horse diseases, with mortality rates of up to 95\% (Coetzer and Guthrie, 2004). Orbiviruses are non-enveloped viruses with a double-layered protein capsid and a segmented double-stranded (ds) RNA genome (Mertens and Diprose, 2004; Roy et al., 1994). The complete viral replication cycle takes place in the cytoplasm of infected cells. Once a virus has entered a host cell via endocytosis, its outer protein coat is removed. The core particle is released into the cytoplasm, the viral genome transcribed and translated, and progeny virions assembled in large granular cytoplasmic regions called virus inclusion bodies. From there they are trafficked to the plasma membrane for virus release. Release can occur either by localised membrane destruction and cell lysis, or via a budding-type process from the plasma membrane whereby the virus acquires a transient membrane envelope that is lost soon after release (Beaton et al., 2002; Han and Harty, 2004; Owens et al., 2004; Stoltz et al., 1996; Wirblich et al., 2006). We recently compared different AHSV strains for their effects on infected mammalian cells, and identified major inter-strain differences in terms of the resulting cytopathic effect (CPE), cell viability, membrane permeability and virus release (Meiring et al., 2009). We are now interested in characterising these phenomena on an ultrastructural level, specifically in investigating whether the predominating mechanisms of virus release (lytic vs budding) could be one of the factors contributing to differences in the observed degree of cell death.

We compared different methods of culturing and processing an adherent mammalian cell line infected with AHSV for cryoimmobilization by HPF-FS and analysis by TEM. We were specifically interested in studying associations between viruses and the plasma membrane in non-trypsinized and mechanically unstressed cells preserved in their native state. We identified a novel way of culturing and infecting cells on the inner surface of cellulose microcapillary tubes, obtained significant benefits in comparison to other methods commonly employed, and demonstrated the success of this method as a model for viral infections and monitoring of viral release events. 


\section{MATERIALS AND METHODS}

\section{Culturing of cells}

Vero cells (ATCC CCL-81) were cultured in the presence of MEM (Eagle's Minimal Essential Medium) containing $5 \%$ foetal calf serum, non-essential amino acids and antibiotics and antifungals (penicillin, streptomycin and fungizone) at $37^{\circ} \mathrm{C}$ in $5 \% \mathrm{CO}_{2}$. Vero cells are routinely cultured as a monolayer in a tissue culture dish. For the purpose of this study the cells were either grown directly in the dish, or cultured upon two different support layers, i.e. small sapphire disks or Aclar membranes, or alternatively grown inside cellulose microcapillary tubes. Sterilised sapphire disks (LEICA Supplies) or $1.2 \mathrm{~mm}$ diameter circles punched from Aclar sheets (SPI Supplies) were positioned in the tissue culture dish prior to seeding of the cells (Jimenez et al., 2006; Reipert et al., 2004). Cells were then seeded and left for 48 hours to achieve sufficient confluency. For growing cells inside capillary tubes, the Vero cells were dislodged by trypsination and allowed to sediment to form a dense cell suspension. The cellulose microcapillary tubes (LEICA Supplies) with a $200 \mu \mathrm{m}$ inner diameter, were sterilised by UV light for 20 minutes prior to use, and handled under sterile conditions throughout cell culturing. The tubes were cut to lengths of $3 \mathrm{~cm}$, and carefully handled with fine forceps. The tip of a tube was inserted into the concentrated cell suspension, and the cells filled the tubes automatically by capillary action (Hohenberg et al., 1994). Cells were left to attach by placing the tubes horizontally in a tissue culture dish, submerged in medium. Rotating the tube during the first 4 hours of attachment facilitated growth of the cells around the full inner surface of the tube. Medium within the tubes was replaced manually at regular intervals over a three day period. This was done by holding the tube in a vertical position with the lower end of the tube immersed in growth medium, and applying filter paper to the top end of the tube. This capillary action allowed the exchange of medium in the tube with fresh medium from an external source, and dislodged and removed any unattached cells. Cell growth was monitored by light microscopy until cells reached approximately $90 \%$ confluency. The number of cells attached to the inner surface of the tube was calculated to be $\pm 2.8 \times 10^{4}$ cells, 
based on the assumed density of $1 \times 10^{5}-2.5 \times 10^{5}$ Vero cells per $\mathrm{cm}^{2}$ at confluency (www.atcc.org).

\section{Virus infection}

AHSV serotype 3 (AHSV-3) was obtained from the OIE Reference Laboratory at the Onderstepoort Veterinary Institute (OVI), South Africa. Cells were rinsed with serumfree medium and subsequently infected with virus at a multiplicity of infection (MOI) of at least 2 plaque-forming units (pfu) per cell. Viruses were allowed to attach for a period of 1 hour at $37^{\circ} \mathrm{C}$, after which the inoculum was removed and replaced with fresh serumfree medium. For cells grown inside microcapillaries, the virus infection was done using the inherent capillary action of the tubes as described above, where one end of the tube was inserted into virus inoculum rather than medium. In order to achieve the desired $\mathrm{MOI}$, the residual volume inside the tube following confluent cell growth was calculated to be $\pm 0.6 \mu \mathrm{l}$, based on the observation that the Vero cell layer did not exceed $20 \mu \mathrm{m}$, leaving a minimum residual $160 \mu \mathrm{m}$ inner diameter. Thus when the tube was placed into the virus inoculum, $\pm 0.6 \mu \mathrm{l}$ of inoculum at an average virus titre of $5 \times 10^{8}$ viruses $/ \mathrm{ml}$ would be sufficient to reach an $\mathrm{MOI}$ of at least 10 , which would ensure a synchronised infection of all cells. Again the inoculum was removed after an hour of virus adsorption and replaced with medium. Infected cells were incubated at $37^{\circ} \mathrm{C}$ for 24 to 48 hours before processing, while control samples were removed one hour post infection.

\section{High-pressure freezing and freeze-substitution}

High-pressure freezing was done with the use of the Leica EMPACT2 HPF apparatus following a standardised protocol (Studer et al., 2001), with some modifications depending on the method used to culture and/or harvest the cells. Cells grown in a tissue culture dish were gently dislodged by scraping, and concentrated by low speed centrifugation (1000 rpm for 5 minutes) into a loose pellet. A volume of $0.5 \mu$ of the cell pellet was transferred by micropipette into the Leica membrane specimen carrier. For cells grown on sapphire or Aclar discs, the support layers were loaded into a specimen carrier and special care taken to keep them in the right orientation, with cells facing upwards. The cellulose microcapillary tubes were cut into 1-2 mm pieces with a sharp 
blade while keeping them submerged in medium, and the ends sealed with a blunt knife. The microcapillary tube segments were then loaded into a specimen carrier. All samples were overlaid with the cryoprotectant 1-hexadecane, and frozen within $10 \mathrm{~ms}$ to the temperature of liquid nitrogen $\left(-196^{\circ} \mathrm{C}\right)$ and pressurized to 2000 bar with methylcyclohexane as hydraulic fluid (Studer et al., 2001). Following HPF, freeze substitution (FS) was done in a LEICA AFS2 FS machine with a FS mixture of acetone containing $1 \%$ osmium tetraoxide and $1 \%$ water. During the FS procedure, the temperature was increased logarithmically from $-90^{\circ} \mathrm{C}$ to $0^{\circ} \mathrm{C}$ over a period of 72 hours. The units were then washed 3 times with clean acetone.

Resin embedding, ultrathin sectioning, staining and TEM viewing

Embedding was done with a hard resin formulation, Quetol 651 epoxy resin (Van der Merwe and Coetzee, 1992), and ultrathin sections $(100 \mathrm{~nm})$ were made with a Reichert Jung Ultracut E microtome with a Diatome diamond knife. For cells that had been removed from the tissue culture dish prior to HPF, ultrathin sections were made through the cell pellet. For the cells cultured upon the sapphire disks and Aclar films, the support layers had to be separated from the embedded cells prior to sectioning. After completely exposing the sapphire disc, it was removed by rapidly freezing the disc in liquid nitrogen and then heating the disc on a heating block. For samples grown on Aclar film, fine forceps were used to remove the film from the resin upon exposure. In both cases the cells remained behind within the resin. Following removal of the support layers, microtome sections were made through the exposed cells parallel to the plane of the monolayer within the resin. Microcapillary tubes did not have to be removed prior to sectioning and could be treated as pieces of tissue, and sections were made either parallel or perpendicular to the length of the tubes. Multiple microcapillary tubes were embedded next to each other to increase the amount of material available for study. Ultrathin sections were stained for 15 min in 1\% uranyl acetate and 3 min in Reynolds' lead citrate (Reynolds, 1963), followed by TEM visualisation with a JEOL JEM-2100F field emission transmission electron microscope. 


\section{RESULTS AND DISCUSSION}

We are interested in studying the AHSV life cycle and cellular pathogenesis, especially from the viewpoint of comparing different virulent and non-virulent AHSV strains and their effects on different cell types. The current postulate is that in mammalian cells lytic virus release predominates and leads to severe CPE, whereas in insect cell cultures that lack discernible CPE virus release is mainly by nonlytic budding-type events. The mechanisms that underlie and regulate these different types of virus release are not known. Recently we evaluated a panel of viruses which showed differences in the CPE they induced in Vero cells (Meiring et al., 2009), and we set out to investigate this on an ultrastructural level. As high quality preservation of specifically membranous structures would be crucial for studying virus mediated membrane release events, all samples were prepared by HPF-FS.
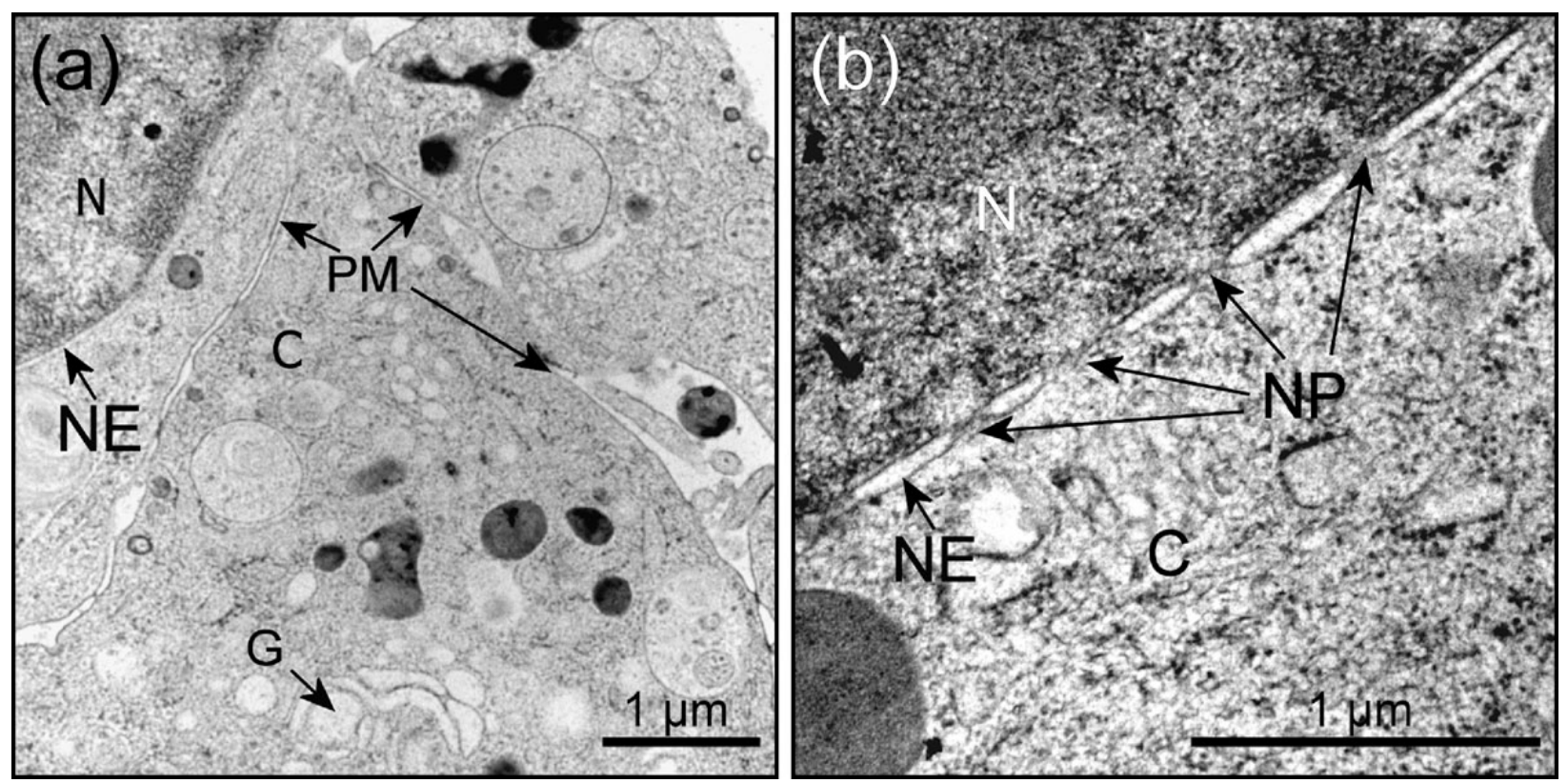

Fig. 1. TEM images of Vero cells following sample preparation from a cell pellet by HPF-FS. Neighbouring cells (a) show excellent preservation of membranous structures such as the plasma membranes (PM), nuclear envelope (NE) and Golgi apparatus (G). In (b) the presence of multiple nuclear pores (NP) are evident within the double-layered nuclear envelope (NE) separating the nucleus (N) from the cytoplasm (C). 
Cells were initially harvested by gently dislodging them from the tissue culture dish, collected by centrifugation and processed from a cell pellet. A good level of preservation of ultrastructure was present (Fig 1), as evident from the density of the cytoplasm, the intact plasma membranes, double-layered nuclear envelopes, nuclear pores and well preserved membranous structures such as the Golgi apparatus. Following AHSV infection, high resolution images of virus related structures and events were obtained (Fig 2). The most striking feature of virus infected cells was the presence of large rod-shaped crystalline structures (Fig 2a, b) in the nucleus and/or cytoplasm. These are similar in appearance to the "intracellular inclusions" previously described (Breese et al., 1969) and probably represent the VP7 crystals that can be purified from AHSV infected cells (Burroughs et al., 1994). These crystals are unique to AHSV, and have not been observed for any other orbiviruses. Characteristic however of all orbivirus infections is the appearance of virus encoded cytoplasmic tubules early in infection (Huismans and Els, 1979). The tubules are formed by the polymerisation of the viral protein NS1, and in the case of AHSV they are of varying length with a diameter of approximately $23 \mathrm{~nm}$ (Maree and Huismans, 1997). The function of the NS1 tubules remains unknown, but large numbers of them were detected in the cytoplasm (Fig 2b, c). Cytoplasmic viruses (70 nm spherical structures with an electron dense core) were present either as free particles, as small aggregates (Fig 2b), as paracrystalline arrays (Fig $2 \mathrm{c}$ ) or within the grainy matrix of the viral inclusion bodies (VIB) (Fig 2d). These are cytoplasmic bodies composed of a NS2 protein matrix that forms a scaffold for virus packaging and assembly (Kar et al., 2007; Uitenweerde et al., 1995).

All of these structures and events confirmed successful AHSV infection. We however observed a distortion of the shape of the cells due to their mechanical removal. This was especially evident in infected cells when compared to uninfected controls (not shown), where the typical elongated Vero cells now appeared rounded. Loss of plasma membrane integrity could be observed in infected cells (Fig $2 \mathrm{~b}$ ), more so at later stages post infection, where up to $40 \%$ of cells had damaged cell membranes We were specifically interested in virus release, but very rarely succeeded in capturing an image 
of a release event - irrespective of whether it was of a lytic nature or by budding. In
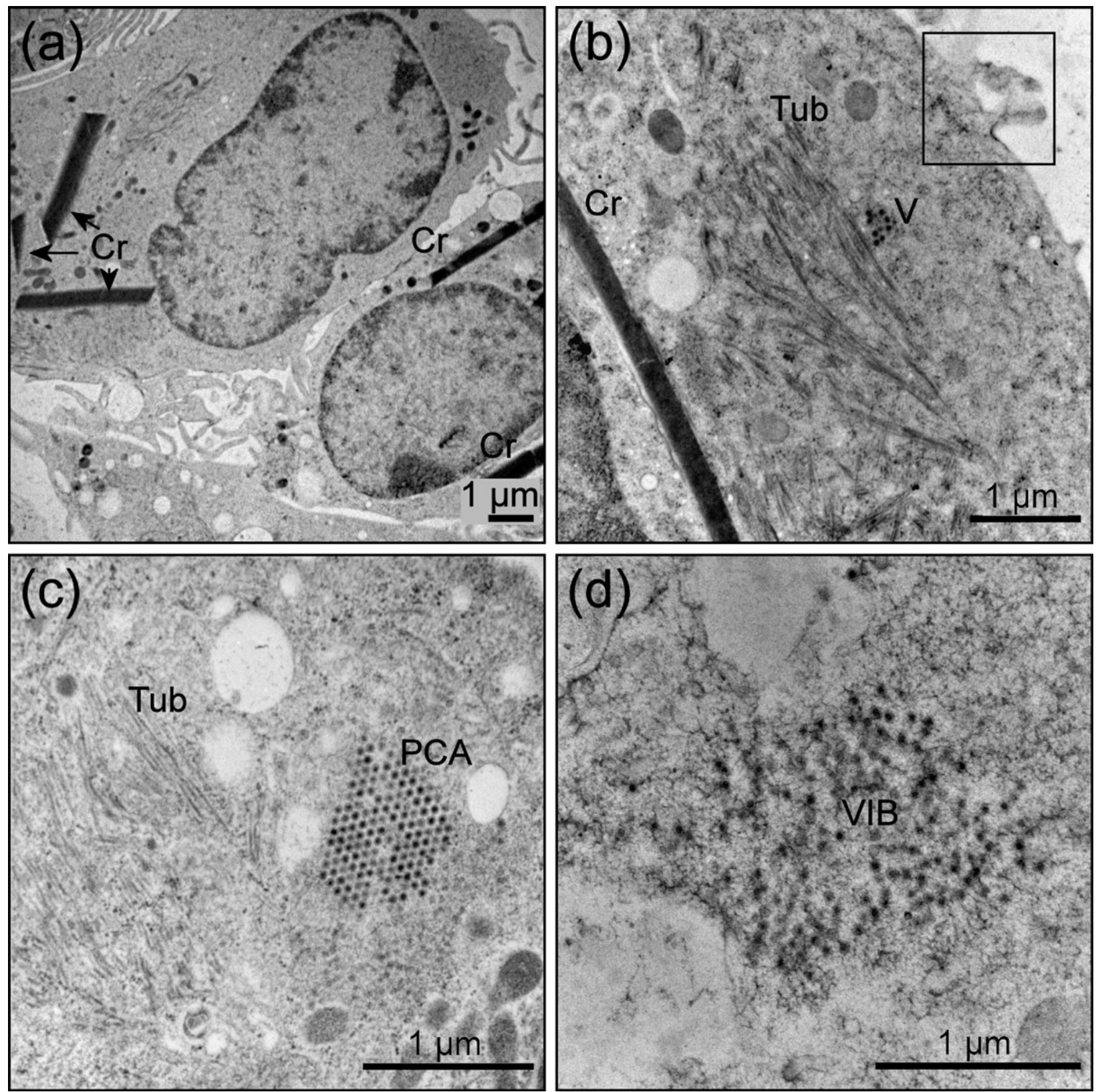

Fig. 2. TEM images of AHSV infected Vero cells following sample preparation from a cell pellet. An area of neighbouring cells (a), as well as single cells ( $b, c$ and d) with evidence of infection such as rod-shaped crystalline structures (Cr), NS1 tubules (Tub), AHSV virions (V), a viral para-crystalline array (PCA), and NS2 viral inclusion bodies (VIB). The block in (b) shows a disrupted cell membrane.

more than 1500 cells examined, only two incidences of active non-lytic viral release were observed. We postulated that the areas of plasma membrane damage could be the result of lytic release, but again in most cases we were not able to confirm this 
based the presence of virus particles directly associated with remnants of cellular membranes at these sites. Theoretically the damage could also arise from physical manipulation of cells with virus-induced increased membrane permeability or compromised membrane integrity (Beaton et al., 2002; Han and Harty, 2004; Owens et al., 2004; Stoltz et al., 1996; Wirblich et al., 2006). The frequency of release events visualised was therefore too low for any comparative study between different strains. If the handling of the cells, i.e. the removal from their growth surface and centrifugal concentration prior to HPF-FS, dislodged the viruses present or being released at points of membrane damage a non-invasive method for processing cells could prevent this.
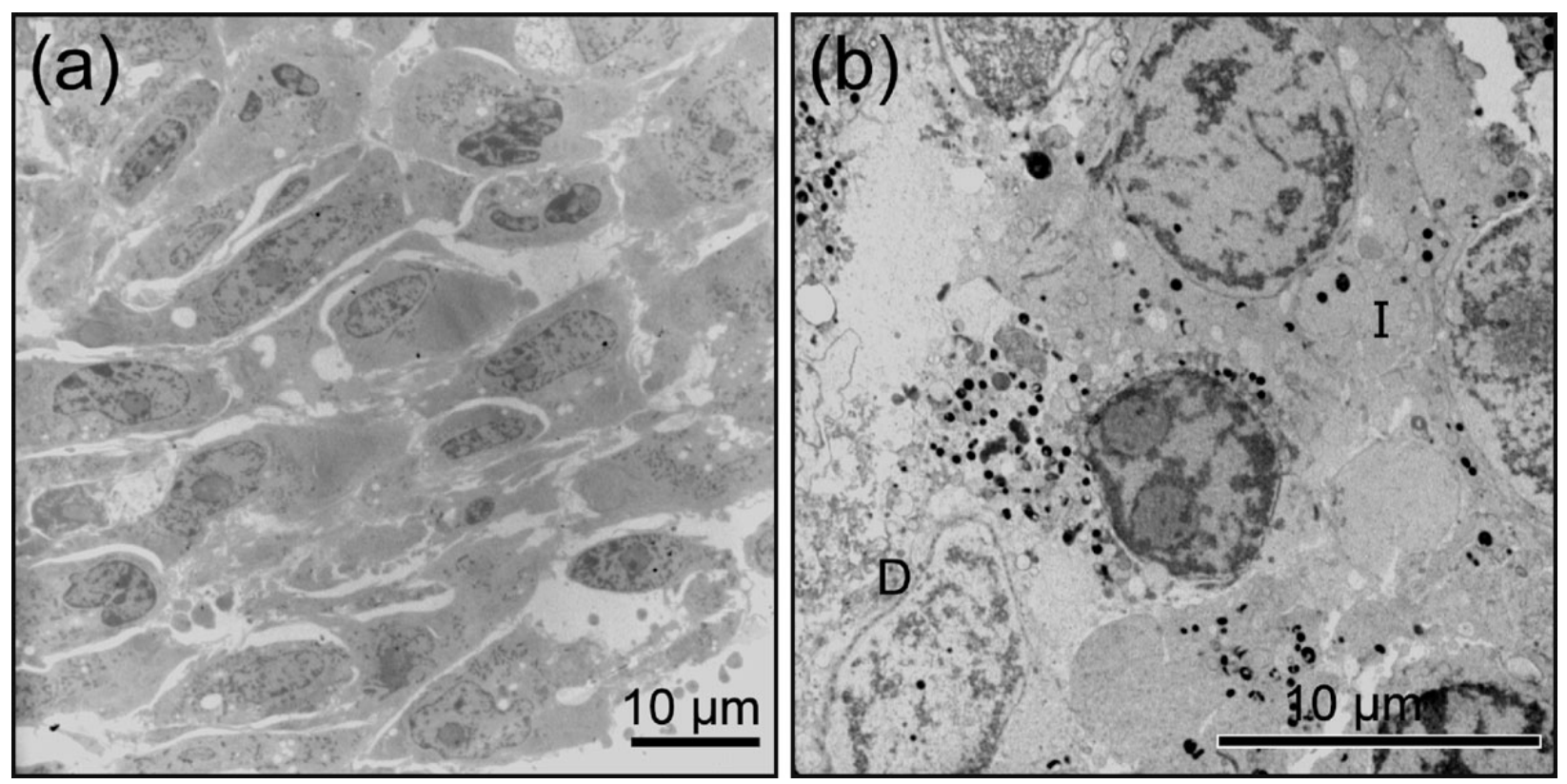

Fig. 3. TEM images of Vero cells grown and processed on different support layers. Noninfected cells cultured upon Aclar films (a) show a field of intact cells. Cells cultured on sapphire discs and infected with AHSV (b) show areas of damaged (D) and intact unaffected (I) neighbouring cells.

First we cultured and processed cells on two support layers previously described as suitable for these types of applications, i.e. sapphire disks (Reipert et al., 2004) and Aclar films (Jimenez et al., 2006). The removal of the support layers from the resin prior to ultrathin sectioning in some cases caused damage to the cellular material, which was unexpected as a hard formulation resin was used. When the Aclar film was not removed before sectioning, tearing of samples at the cell-film interface often occurred. 
Although we could obtain high resolution images of a field of neighbouring cells in their native state from the single layer of cells that remained in the resin (Fig 3a, b), these methods were technically demanding and did not prove to be amenable to generating images of cell surface events.
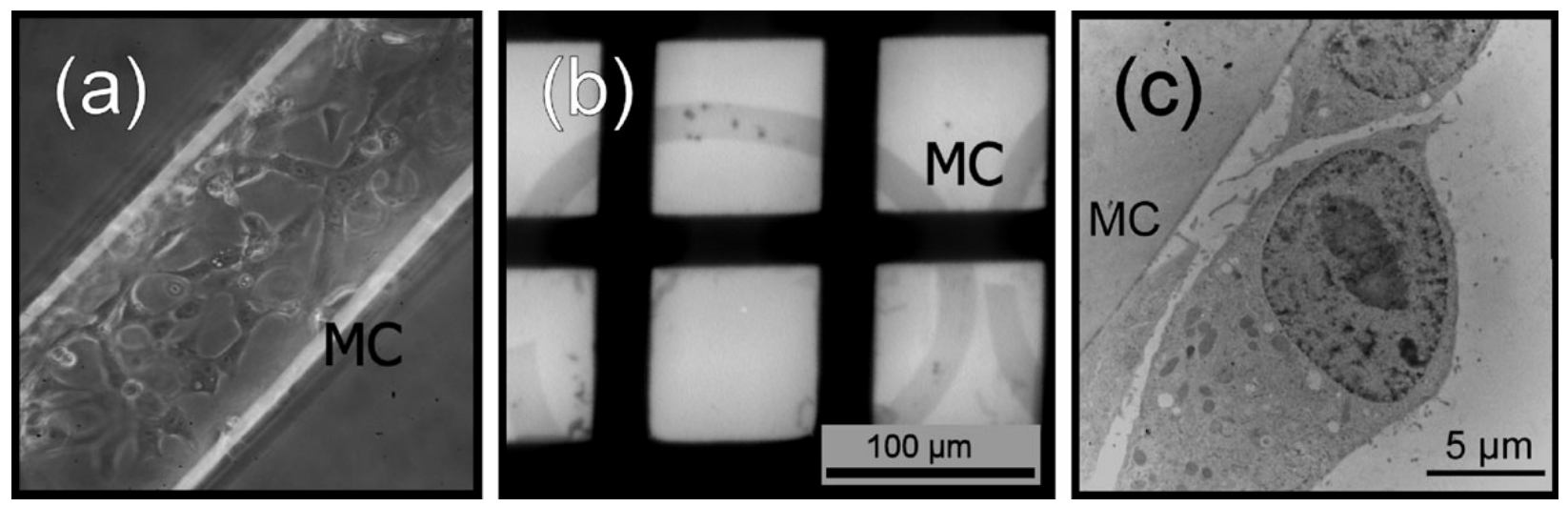

Fig. 4. Culturing of Vero cells inside cellulose microcapillary (MC) tubes. Light microscope image (a) of semi-confluent cells at $24 \mathrm{~h}$ post-seeding. TEM images of cross section through a MC tube at low magnification (b) and at higher magnification that allows identification of single cells attached to the inner wall of the tube (c).

It was therefore decided to investigate culturing cells as a monolayer on the internal surface of cellulose microcapillary tubes. Where microcapillaries were previously described for sample processing by HPF-FS, the cells were either mechanically dislodged and then used to fill the tubes (Hohenberg et al., 1994; Muller-Reichert et al., 2003; Rieger et al., 1997; Studer et al., 2001), or cultured inside the tubes to a high density, completely filling the tubes (Tiedemann et al., 1998). Using an adapted protocol of Hohenberg et al. (1994), cells from a trypsinized suspension were introduced into the tubes by capillary action. From light microscopic monitoring it was established that the Vero cells successfully attached to the capillaries and grew to a semi-confluent monolayer all around the tube's inner surface over a period of 48 to 72 hours (Fig 4a). After HPF-FS processing and embedding the tube sections could easily be identified within the resin, thus facilitating the identification of the potential location of cellular material. In addition, multiple tubes could be embedded next to each other in the same resin block, so that four to ten tube sections were often present on a single EM grid. 
When viewed by TEM it was clear that the tubes had retained their shape (Fig 4b). As we experienced no problems with tube compression, and wanted to minimize any cellular disturbance via fluid movements in the tubes, we opted to not include a filler material in the open cavity as previously suggested (Daghma et al., 2011). Ultrathin sectioning could easily be done through the cellulose, without damaging or detaching the cells, and a layer of intact Vero cells was visible on the inner surface of the microcapillary wall (Fig 4c). This resulted in perfect maintenance of the orientation of neighbouring cells, with an undisturbed cell surface area available for study.

The next parameter under investigation was to determine whether the Vero cells could be infected with viruses. As the remaining inner diameter was sufficient to allow movement of fluids, introducing the virus inoculum and subsequent replacement with fresh medium could all be done via capillary action. Following processing and TEM visualisation (Fig 5), single cells or an area of neighbouring cells could be visualised, firmly attached to the microcapillary tube surface (Fig 5a, c, e). All cells were in their native state, with no physical distortion. AHSV replication was evident from the presence of previously described virus infection hallmarks, such as viral inclusion bodies (Fig 5, a, c, d, e), crystalline structures (Fig 5c), and individual virus particles present in the cytoplasm. Multiple released viruses could be observed between adjacent intact membranes of infected cells (Fig 5 d). In the order of $90 \%$ of the cell population was infected, on par with what was achieved when traditional tissue culture conditions were used. At 24 hours post infection, approximately $65 \%$ of the cells had viruses localised at the plasma membrane or on the outer cell surface. To ensure that these did not represent residual non-adsorbed viruses from the initial infection, control cells were fixed and processed one hour after the inoculum was removed (not shown). This resulted in only $8 \%$ of cells with viruses outside the cell membranes or between adjacent cells, confirming that the bulk of external viruses at the later times after infection (such as in Fig 5a, $d$ and e) would indeed be newly released progeny virions. We were able to capture images of viruses both in the process of being released via budding, as evident by the presence of a transient membrane (Fig $5 a, b$ ) and events of lytic release (Fig $5 e, f)$ with viruses present at sites of membrane damage. 

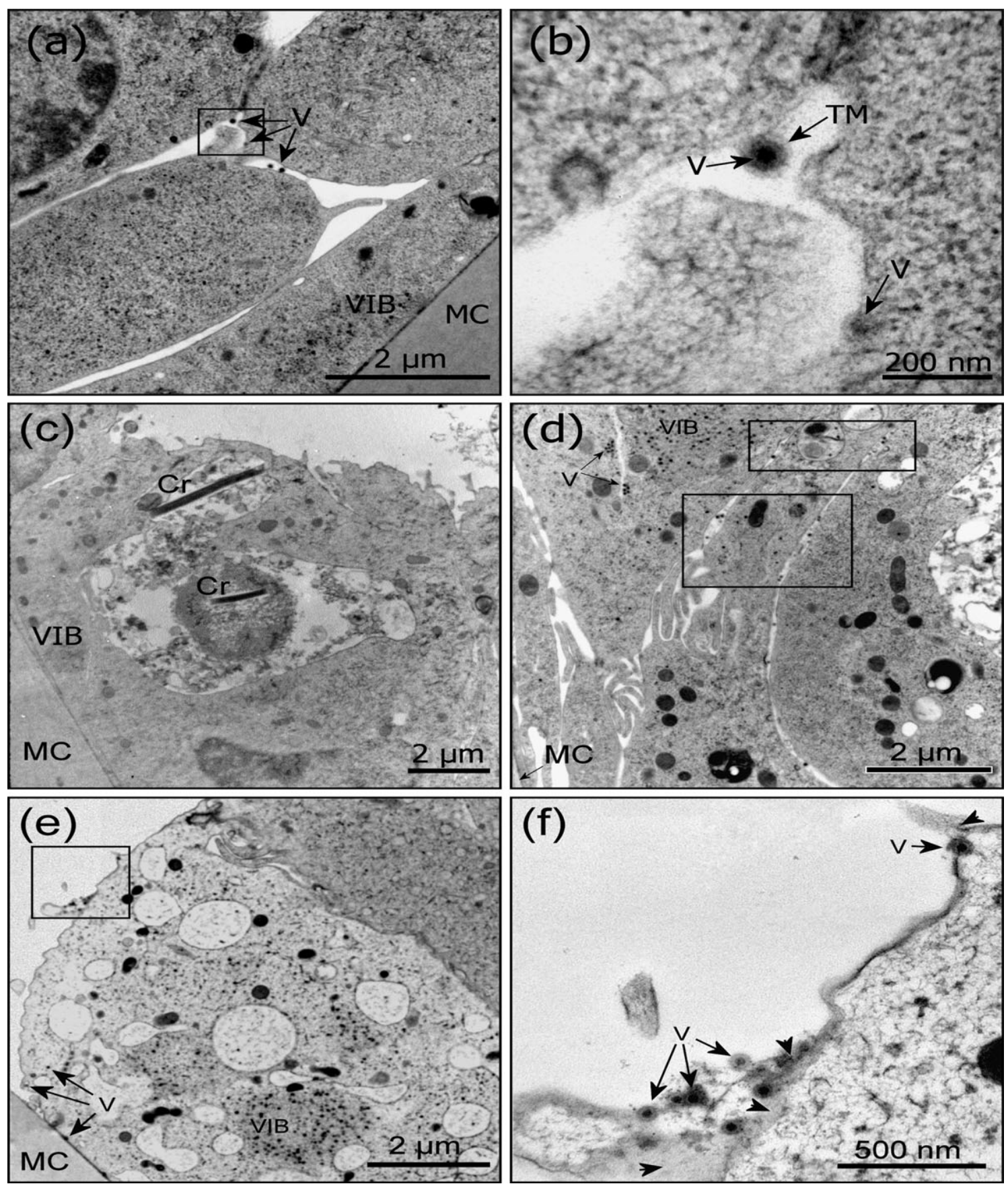

Fig. 5. TEM images of AHSV infected Vero cells grown and processed in microcapillary (MC) tubes. Virus $(V)$ release can be observed at the plasma membrane $(a, b, d$, e and $f)$, and infection related structures such as viral inclusion bodies (VIB) (a, c, d and e) and rod-shaped crystalline structures (Cr) (c) are indicated. The image in (b) is an enlargement of the area indicated in (a) and shows a non-lytic form of release as evident by the transient membrane (TM) surrounding the virus particle. Blocks in (d) indicate released virus particles between adjacent infected cells with intact membranes, demonstrating the occurrence of non-lytic release events. The image in (f) in an enlargement of the area indicated in (e) and shows lytic release with damaged membranes (arrowheads) at sites of release. 
These results confirm that AHSV utilises two modes of release from mammalian cells, and that processing cells inside microcapillary tubes enabled us to view these events at a much higher frequency than when cells were harvested and concentrated by centrifugation. This will now allow easy comparison of the subcellular localisation and release of strains of AHSV with different pathogenicities. This novel system described here could be utilised for studies of any virus that can infect an adherent cell line, but is especially suited to viruses that give relatively low yields in tissue culture. When compared to other members of the Reoviridae family, such as bluetongue virus (BTV) or reovirus, AHSV replicates to log-fold lower titres. Standard TEM processing of BTV infected mammalian cells is sufficient for routine visualisation of virus release by budding (Celma and Roy, 2009). No BTV budding from the plasma membrane has however been captured in insect cells at any time point, even though release from insect cells would appear to be primarily nonlytic based on the absence of any observed cytopathic effect following infection (Celma and Roy, 2011). We have recently managed to also successfully culture an insect cell line (KC cells, from Culicoides) in the cellulose microcapillary tubes. This will facilitate future studies of both BTV and AHSV release from cells derived from their insect vector.

\section{ACKNOWLEDGEMENTS}

The work was funded by the University of Pretoria, the National Research Foundation and the Microscopy Society of Southern Africa Trust. We would like to thank Mr Flip Wege for valuable technical assistance and Prof Henk Huismans for critical reading of the manuscript. The author and co-authors have no conflict of interest to declare.

\section{REFERENCES}

Beaton AR, Rodriguez J, Reddy YK, Roy P. 2002. The membrane trafficking protein calpactin forms a complex with bluetongue virus protein NS3 and mediates virus release. Proc Natl Acad Sci U S A 99(20):13154-13159. 
Breese SS, Jr., Ozawa Y, Dardiri AH. 1969. Electron microscopic characterization of African horse-sickness virus. J Am Vet Med Assoc 155(2):391-400.

Burroughs JN, O'Hara RS, Smale CJ, Hamblin C, Walton A, Armstrong R, Mertens PP. 1994. Purification and properties of virus particles, infectious subviral particles, cores and VP7 crystals of African horsesickness virus serotype 9. J Gen Virol 75 (Pt 8):1849-1857.

Celma CC, Roy P. 2009. A viral nonstructural protein regulates bluetongue virus trafficking and release. J Virol 83(13):6806-6816.

Celma CC, Roy P. 2011. Interaction of calpactin light chain (S100A10/p11) and a viral NS protein is essential for intracellular trafficking of nonenveloped bluetongue virus. J Virol 85(10):4783-4791.

Coetzer JAW, Guthrie AJ. 2004. African Horse sickness. In Infectious Diseases of Livestock. Cape Town: Oxford University Press Southern Africa. 1231-1246 p.

Daghma DS, Kumlehn J, Melzer M. 2011. The use of cyanobacteria as filler in nitrocellulose capillaries improves ultrastructural preservation of immature barley pollen upon high pressure freezing. J Microsc 244(1):79-84.

Fontana J, López-Iglesias C, Tzeng W-P, Frey TK, Fernández JJ, Risco C. 2010. Three-dimensional structure of Rubella virus factories. Virology 405(2):579-591.

Hagen C, Grünewald K. 2008. Microcarriers for high-pressure freezing and cryosectioning of adherent cells. J Microsc 230(Pt 2):288-296.

Han Z, Harty RN. 2004. The NS3 protein of bluetongue virus exhibits viroporin-like properties. J Biol Chem 279(41):43092-43097.

Hohenberg H, Mannweiler K, Muller M. 1994. High-pressure freezing of cell suspensions in cellulose capillary tubes. J Microsc 175(Pt 1):34-43.

Huismans H, Els HJ. 1979. Characterization of the tubules associated with the replication of three different orbiviruses. Virology 92(2):397-406.

Jimenez N, Humbel BM, van Donselaar E, Verkleij AJ, Burger KN. 2006. Aclar discs: a versatile substrate for routine high-pressure freezing of mammalian cell monolayers. J Microsc 221(Pt 3):216-223.

Kar AK, Bhattacharya B, Roy P. 2007. Bluetongue virus RNA binding protein NS2 is a modulator of viral replication and assembly. BMC Mol Biol 8:4. 
Maree FF, Huismans H. 1997. Characterization of tubular structures composed of nonstructural protein NS1 of African horsesickness virus expressed in insect cells. J Gen Virol 78 (Pt 5):1077-1082.

Meiring TL, Huismans H, van Staden V. 2009. Genome segment reassortment identifies non-structural protein NS3 as a key protein in African horsesickness virus release and alteration of membrane permeability. Arch Virol 154(2):263-271.

Mertens PP, Diprose J. 2004. The bluetongue virus core: a nano-scale transcription machine. Virus Res 101(1):29-43.

Monaghan P, Cook H, Jackson T, Ryan M, Wileman T. 2004. The ultrastructure of the developing replication site in foot-and-mouth disease virus-infected BHK-38 cells. J Gen Virol 85(Pt 4):933-946.

Morphew MK, Mclntosh JR. 2003. The use of filter membranes for high-pressure freezing of cell monolayers. J Microsc 212(Pt 1):21-25.

Muller-Reichert T, Hohenberg H, O'Toole ET, McDonald K. 2003. Cryoimmobilization and three-dimensional visualization of C. elegans ultrastructure. J Microsc 212(Pt 1):71-80.

Owens RJ, Limn C, Roy P. 2004. Role of an arbovirus nonstructural protein in cellular pathogenesis and virus release. J Virol 78(12):6649-6656.

Reichelt M, Li W, Sommer M, Perrino J, Nour AM, Sen N, Baiker A, Zerboni L, Arvin AM. 2011. Entrapment of Viral Capsids in Nuclear PML Cages Is an Intrinsic Antiviral Host Defense against Varicella-Zoster Virus. PLoS Pathogens 7(2):1-20.

Reipert S, Fischer I, Wiche G. 2004. High-pressure freezing of epithelial cells on sapphire coverslips. J Microsc 213(Pt 1):81-85.

Reynolds ES. 1963. The use of lead citrate at high $\mathrm{pH}$ as an electron-opaque stain in electron microscopy. J Cell Biol 17:208-212.

Rieger G, Müller K, Hermann R, Stetter KO, Rachel R. 1997. Cultivation of hyperthermophilic archaea in capillary tubes resulting in improved preservation of fine structures. Archives of Microbiology 168(5):373-379.

Roy P, Mertens PP, Casal I. 1994. African horse sickness virus structure. Comp Immunol Microbiol Infect Dis 17(3-4):243-273. 
Stoltz MA, van der Merwe CF, Coetzee J, Huismans H. 1996. Subcellular localization of the nonstructural protein NS3 of African horsesickness virus. Onderstepoort J Vet Res 63(1):57-61.

Studer D, Graber W, Al-Amoudi A, Eggli P. 2001. A new approach for cryofixation by high-pressure freezing. J Microsc 203(Pt 3):285-294.

Studer D, Hennecke H, Müller M. 1992. High-pressure freezing of soybean nodules leads to an improved preservation of ultrastructure. Planta 188(2):155-163.

Suhy DA, Giddings TH, Jr., Kirkegaard K. 2000. Remodeling the endoplasmic reticulum by poliovirus infection and by individual viral proteins: an autophagy-like origin for virus-induced vesicles. J Virol 74(19):8953-8965.

Tiedemann, Hohenberg, Kollmann. 1998. High-pressure freezing of plant cells cultured in cellulose microcapillaries. Journal of Microscopy 189(2):163-171.

Uitenweerde JM, Theron J, Stoltz MA, Huismans H. 1995. The Multimeric Nonstructural NS2 Proteins of Bluetongue Virus, African Horsesickness Virus, and Epizootic Hemorrhagic Disease Virus Differ in Their Single-Stranded RNA-Binding Ability. Virology 209(2):624-632.

Van der Merwe CF, Coetzee J. 1992. Quetol 651 for general use: a revised formulation. Electron microscopy society of southern Africa 22:31-32.

Wild P, Schraner EM, Cantieni D, Loepfe E, Walther P, Muller M, Engels M. 2002. The significance of the Golgi complex in envelopment of bovine herpesvirus 1 (BHV1) as revealed by cryobased electron microscopy. Micron 33(4):327-337.

Wirblich C, Bhattacharya B, Roy P. 2006. Nonstructural protein 3 of bluetongue virus assists virus release by recruiting ESCRT-I protein Tsg101. J Virol 80(1):460473. 